\title{
OPERATIONS ON RESOLUTIONS AND THE REVERSE ADAMS SPECTRAL SEQUENCE
}

\author{
DAVID BLANC
}

\begin{abstract}
We describe certain operations on resolutions in abelian categories, and apply them to calculate part of a reverse Adams spectral sequence, going "from homotopy to homology", for the space $\mathbf{K}(\mathbb{Z} / 2, n)$. This calculation is then used to deduce that there is no space whose homotopy groups are the reduction mod 2 of $\pi_{*} \mathbf{S}^{r}$.

As another application of the operations we give a short proof of T. Y. Lin's theorem on the infinite projective dimension of all nonfree $\boldsymbol{\pi}$-modules.
\end{abstract}

\section{INTRODUCTION}

In [B1] we described a Hurewicz spectral sequence going "from homotopy to homology": that is, for any connected space $\mathbf{X}$ and abelian group $G$, a spectral sequence converging to $\tilde{H}_{*}(\mathbf{X} ; G)$, whose $E^{2}$-term is isomorphic to certain derived functors of the $\Pi$-algebra of $\mathbf{X}$-i.e., of the graded group $\pi_{*} \mathbf{X}$, together with the action of the primary homotopy operations on it (cf. [B1, $\S 3.1 .1]$ or $[S, \S 4])$.

To illustrate the operation of the spectral sequence, we here calculate the $E^{2}$ term and differentials for $\mathbf{X}=\mathbf{K}(\mathbb{Z} / 2, n)$. This particular case (which may be termed the reverse Adams spectral sequence) has some interest in its own right; for example, it may be be used to deduce the following

Proposition 4.3.6. There is no map $f: \mathbf{S}^{r} \rightarrow \mathbf{X}$ which induces reduction $\bmod 2$ on homotopy groups-in other words, there is no space $\mathbf{X}$ such that $\pi_{*} \mathbf{X} \cong$ $\pi_{*} \mathbf{S}^{r} \otimes \mathbb{Z} / 2$ as $\Pi$-algebras (for $r \geq 6$ ).

Our main tool in this caculation are certain (noncanonical) operations on resolutions in abelian categories, defined in $\S 2$. These are used to calculate part of the $E^{2}$-term for the spectral sequence (in $\S 3$ ), and turn out to be related in our case to the action of the Steenrod algebra $(\S 4.3)$. We also show how $d^{2}$ may be expressed in terms of Toda brackets (Proposition 4.2.4), actually calculate this differential in a certain range (Proposition 4.2.5), and obtain the $E^{\infty}$-term in this range (Proposition 4.3.5).

Received by the editors July 11, 1990 and, in revised form, November 21, 1991.

1991 Mathematics Subject Classification. Primary 55T99; Secondary 18G10.

Key words and phrases. Resolutions, operations, homology, homotopy groups, П-algebras, $\pi$ modules, spectral sequences. 
As another application of these operations, in $\S 5$ we give a short proof of T. Y. Lin's theorem [L1, L2], which states that any $\pi$-module (of finite type) which is not free has infinite projective dimension.

\section{OPERATIONS ON RESOlUtions}

In this section we describe some general facts about resolutions of modules over a ring, showing how they may be constructed so as to support the action of a certain module of operators (depending on the ring).

2.1. Ingredients needed. Let $R$ be a ring with unit, and consider the category of (left) $R$-modules. Given an $R$-module $X$, we wish to construct a free resolution $P_{*}$ of $X$.

2.1.1. A presentation for $X$. For any set $S$, let $R\langle S\rangle$ denote the free $R$-module generated by $S$. In order to construct a (noncanonical) resolution in the usual way, we start with a presentation for $X$ : that is, choose a set of generators $S_{0}$ for $X$, let $P_{0}=R\left\langle S_{0}\right\rangle$, (with the obvious augmentation $\partial_{0}: P_{0} \rightarrow X$ ); choose a set $S_{1}$ of generators for $Z_{0}=\operatorname{Ker}\left(\partial_{0}\right)$, and let $P_{1}=R\left\langle S_{1}\right\rangle$, with the obvious $\partial_{1}: P_{1} \rightarrow P_{0}$.

By repeated choices of this kind one could construct a full resolution $P_{*} \rightarrow X$. However, note that $X$ is in fact determined by the sets $S_{0}$ and $S_{1} \subseteq R\left\langle S_{0}\right\rangle$. Thus, one should be able to construct a resolution out of $S_{0}, S_{1} \subseteq R\left\langle S_{0}\right\rangle$, and "general information" about the category of $R$-modules.

2.1.2. The module of relations. Write $B_{0}$ for $R$ considered as a left $R$-module, and $T$ for its underlying set. Let $B_{1}=R\langle T\rangle$. There is an obvious evaluation map ev : $B_{1} \rightarrow B_{0}$; we call $K=\operatorname{Ker}(\mathrm{ev})$ the module of relations for $R$.

2.2. Constructing the resolution. Given sets $S_{0}$ and $S_{1} \subseteq R\left\langle S_{0}\right\rangle$, and the $R$ module $K$ as above, one can construct a resolution $P_{*} \rightarrow X$, along with a preferred basis $S_{n}$ for each free $R$-module $P_{n}$, by induction on $n$ :

2.2.1. The induction step. We assume we are given $i_{m}: S_{m} \subseteq R\left\langle S_{m-1}\right\rangle$ for each $1 \leq m \leq n$, where $P_{m} \cong R\left\langle S_{m}\right\rangle$ and $\partial_{m}$ is induced by $i_{m}$. The elements of $S_{n+1}$ will be generated by a certain action of $K(\S 2.1 .2)$ on $S_{n}$.

2.2.2. The action of $K$. This action (not everywhere defined) may be described as follows: given sums $x=x_{1}+\cdots+x_{k}$, with $x_{i} \in S_{n}$, and $\gamma=\gamma_{1}+\cdots+\gamma_{m}$, with $\gamma_{j} \in K$, the element $\gamma(x) \in R\left\langle S_{n}\right\rangle$ is defined whenever there are elements $a_{1}, \ldots, a_{m}$ in $S_{n-1}$ such that each $x_{i}$ has the form $x_{i}=\sum_{j=1}^{m} r_{i j}\left[a_{j}\right]$, with $r_{i j} \in R$, and elements $s_{i} \in R$ such that each $\gamma_{j}=\sum_{i=1}^{k} s_{i}\left[r_{i j}\right]$. Then

$$
\gamma(x)=\left(\gamma_{1}+\cdots+\gamma_{m}\right)\left(x_{1}+\cdots+x_{k}\right) \text {. }
$$

For example, if $x=r[a]$, (where $x \in R, a \in S_{n-1}$ ), and $\gamma=s[r]$, (so $s r=0$ in $R)$, then $\gamma(x)=s[r[a]] \in R\left\langle S_{n}\right\rangle$.

2.2.3. The image of the action. Note that the subset of $R\left\langle S_{n}\right\rangle$ comprising all elements of the form $\gamma(x)$, for $x=x_{1}+\cdots+x_{k}$ and $\gamma=\gamma_{1}+\cdots+\gamma_{m}$ as above, is just the submodule $Z_{n}=\operatorname{Ker}\left(\partial_{n}\right) \subseteq P_{n}$; so if we choose $S_{n+1}$ to be a set of $R$-module generators for this submodule, we may set $P_{n+1}=R\left\langle S_{n+1}\right\rangle$ to complete the induction step. 
2.3. The example of $\pi_{*}$-modules. We would like to consider the 2-primary stable homotopy ring $\boldsymbol{\pi}=\pi_{*}^{s}\left(\mathbf{S}^{0} ; 2\right)$. However, in order to get a complete description we consider instead its algebraic 6-skeleton (cf. [B2, §4.2]) -that is, $R=\pi^{(6)}$ is the augmented graded $\mathbb{Z}$-algebra generated by $\eta$ and $\nu$ (in dimension 1 and 3 resp.), subject to the relations $2 \eta=0,4 \nu=\eta^{3}, \eta \nu=\nu \eta=0$, and $2 \nu^{2}=0$.

2.3.1. Convention on notation. It is convenient to use a a single lower case Latin letter to represent the generators of $R\langle T\rangle$ (which are themselves elements of $T$, the underlying set of $R$. We shall generally use the letter corresponding to the usual Greek letter, or to a number, and let tildes represent their powers-viz.:

$$
t=[2], \quad h=[\eta], \quad \tilde{h}=\left[\eta^{2}\right], \quad n=[\nu], \quad \tilde{n}=\left[\nu^{2}\right], \ldots .
$$

2.3.2. A description of $K$. Even for this ring the module of relations $K(\S 2.1 .2)$ is quite large; however, for the application we have in mind we need only consider the following elements: $\eta[2], 2[\eta], \nu[\eta], \nu^{2}[2]$; also, the elements arising from the relation $4 \nu=\eta^{3}$ :

$$
A \stackrel{\text { def }}{=}(2 \nu)[2]-\left(\eta^{2}\right)[\eta], \quad B \stackrel{\text { def }}{=} 2[2 \nu]-\left(\eta^{2}\right)[\eta], \quad C \stackrel{\text { def }}{=}(2 \nu)[2]-\eta\left[\eta^{2}\right] ;
$$

and those arising from the commutativity of $R$ :

$$
D \stackrel{\text { def }}{=} 2[\nu]-\nu[2], \quad E \stackrel{\text { def }}{=} \nu\left[\nu^{2}\right]+\nu^{2}[-\nu] .
$$

(There are also relations among the elements of $K-$ e.g., $B-A=2 D-4[\nu]+$ $2[2 \nu]$.

2.3.3. A graphic representation. It will be useful to have a graphic representation for elements $\gamma=\sum_{i=1}^{k} s_{i}\left[r_{i}\right] \in K$, in the form of diagrams as in Figure 1, consisting of:

- an initial vertex (labelled $\gamma$ );

- for each $i$, a vertex $\left[r_{i}\right]$ and an edge from $\gamma$ to it, labelled $s_{i}$;

- a final vertex (labeled by the operand of $\gamma$ ), with an edge labeled $r_{i}$ from the vertex $\left[r_{i}\right]$ to it.

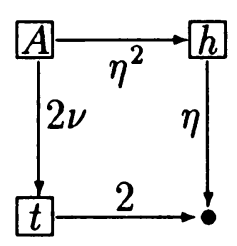

(a)

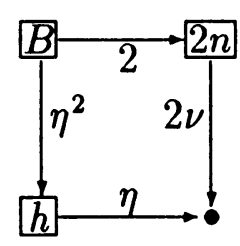

(b)

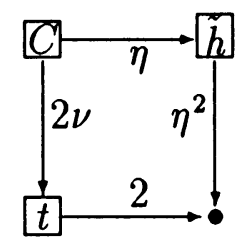

(c)

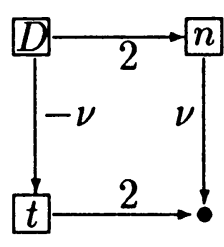

(d)

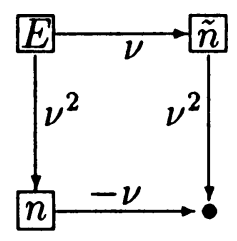

(e)

FiguRE 1. Diagrams for some elements of $K$ 


\section{Calculation for $X=\mathbf{K}(\mathbf{Z} / 2, r)$}

We now wish to apply the construction described in $\S 2$ to the Hurewicz spectral sequence of [B1] for $\mathbf{X}=\mathbf{K}(\mathbb{Z} / 2, r) \quad(r \geq 8)$, in order to calculate the $E^{2}$-term in a certain range.

3.1. Resolving $Z / 2$. Working in a stable "band of dependence" (cf. $\S 4.1$ below), we take the ring $R$ to be $\pi^{(6)}$ (see $\S 2.3$ above), with the $R$-module $X=\pi_{*} X$ equal to $\mathbb{Z} / 2$ concentrated in degree 0 .

In this case we may use the notation of $\S 2.3$ to produce a resolution $P_{*}$ of $X=\mathbb{Z} / 2$ as prescribed in $\S 2$-describing the bases $S_{n} \subseteq R\left\langle S_{n-1}\right\rangle$ for $P_{n}=$ $R\left\langle S_{n}\right\rangle$ in terms of the action of $K(\{2.2 .2)$, where the relevant elements of $K$ were given in $\S 2.3 .2$.

3.1.1. The resolution. We start with a presentation of $X$ (as in $\S 2.1 .1): S_{0}=$ $\{i\}$, where $i$ (in degree 0 ) represents the generator of $X$, and

$$
S_{1}=\{2[i], \eta[i], \nu[i]\} \subset R\left\langle S_{0}\right\rangle
$$

(cf. $\S 2.3 .1)$.

The rest of the resolution is produced by the action of $K$-i.e., the operations of $\S 2.3 .2$ just described-on this presentation. We use the graphic descriptions of $K$ as in Figure 1 above to describe the resolution in Figure 2, with the pattern extended indefinitely by the following

3.1.2. Convention. The set of vertices mapping into a given vertex $x$, in the recursively defined extension of Figure 2, is to be the same as the set of vertices mapping into that vertex $X$ in Figure 2 itself having the same label, and with the same vertices mapping out, as $x$ itself.

This means essentially that every vertex is to have the same set of vertices mapping into it as the circled vertex with the same label.

3.1.3. Note. Note that all vertices with the same name have the same set of arrows mapping in. For example, any vertex marked $t$ (or $t^{\prime}$ ) will have arrows $\eta,-\nu, 2 \nu$ and $\nu^{2}$ mapping into it.

However, the sets of vertices mapping into a given vertex are of two types, (distinguished by a prime $\left({ }^{\prime}\right)$ ): for example, the vertices mapping into plain $t$ will be $h^{\prime}, D, A$, and $\tilde{n}$-as opposed to $h^{\prime}, D, C$, and $\tilde{n}$ mapping into $t^{\prime}$.

3.1.4. Dimensions and degrees. The vertex $i$ represents a free $R$-module on one generator in degree 0 , in homological dimension 0 (i.e., $i \in S_{0}$ ). The homological dimension of every other generator is determined by the length of any (directed) path from it to $i$, while its degree is equal to the sum of the degrees of the elements labeling the edges of such a path.

For example, the circled $A$ is in homological dimension 2 , and in degree $3=|2 \nu|+|2|=\left|\eta^{2}\right|+|\eta|$.

Thus the graph in Figure 2 (suitably continued in all directions) describes the sequence of graded sets $\left\{S_{n}\right\}_{n=0}^{\infty}$, where we collect all generators of homological 


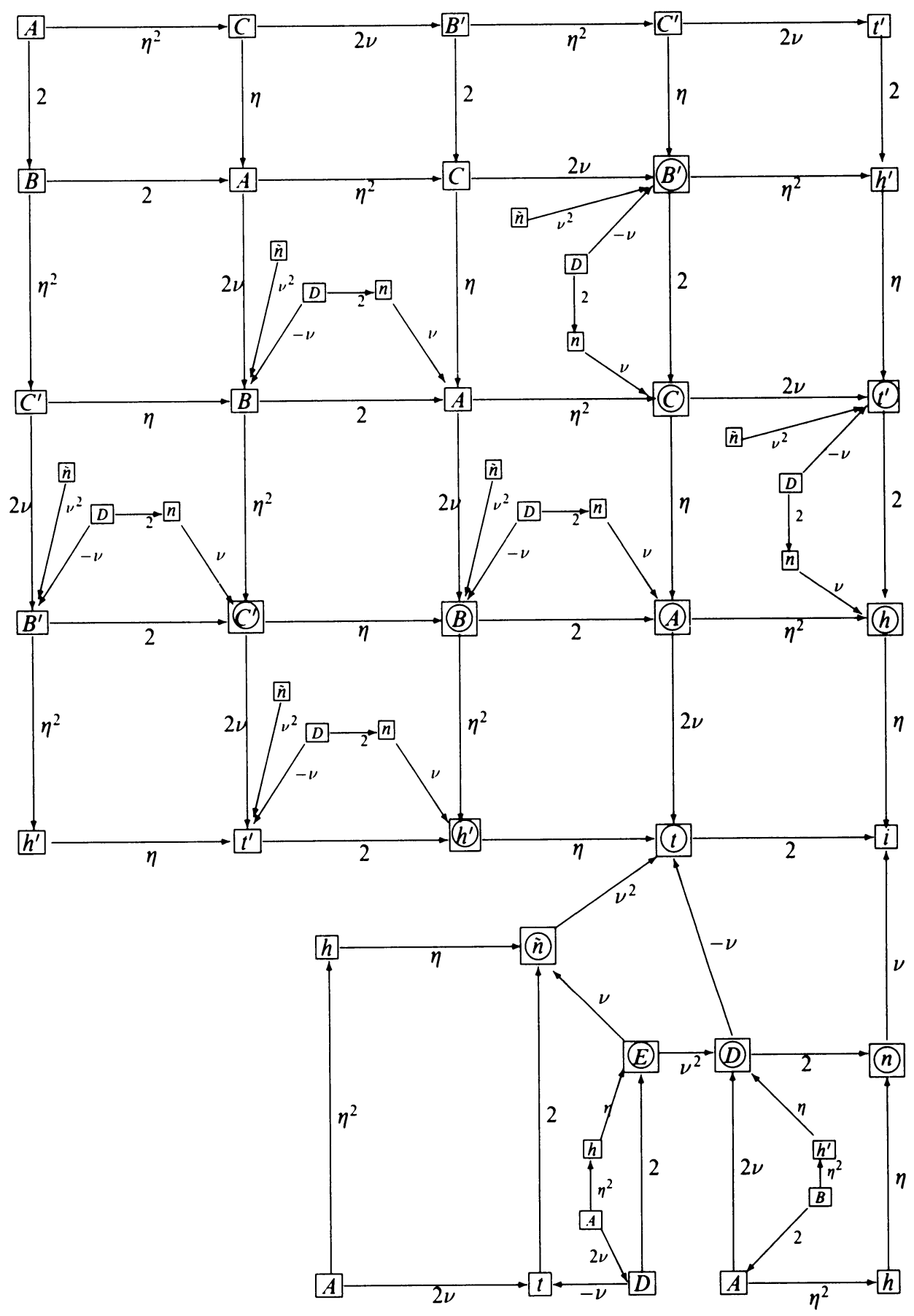

FIGURE 2. A graphic description of the resolution 
degree $n$ in $S_{n}$. Morever, the edges of the graph provide us with maps $i_{n}$ : $S_{n} \rightarrow R\left\langle S_{n-1}\right\rangle$, so that we actually get a chain complex of free graded $R$ modules, which we denote by $P_{*}$.

3.1.5 Proposition. The chain complex $P_{*}$, with the obvious augmentation $P_{0}$ $=R\langle i\rangle \rightarrow X$, constitutes a free $R$-module resolution of $X$.

Proof. The chain complex $P_{*} \rightarrow X$ is clearly acyclic; we must show each $S_{n+1} \subseteq R\left\langle S_{n}\right\rangle$ (described by Figure 2 as in $\S 3.1 .4$ ) constitutes a set of $R$-module generators for $Z_{n}=\operatorname{ker}\left\{\partial_{n}: R\left\langle S_{n}\right\rangle \rightarrow R\left\langle S_{n-1}\right\rangle\right\}$ (cf. $\S 2.2 .3$ ).

If $\gamma=\sum_{i, j} a_{i}\left[b_{i, j}\left[c_{j}\right]\right] \in R\left\langle S_{n}\right\rangle \subseteq R\left\langle R\left\langle S_{n-1}\right\rangle\right\rangle$, then $\partial_{n}(\gamma)=\sum_{i, j} a_{i} b_{i, j}\left[c_{j}\right]$ $\in R\left\langle S_{n-1}\right\rangle$, so $\partial_{n}(\gamma)=0 \Leftrightarrow$ for each $j, \sum_{i, j} a_{i} b_{i, j}=0$.

Thus for each generator $c_{j} \in S_{n-1}$-i.e., for each vertex in Figure 2 which is $n-1$ edges away from $i$-we consider all edges $b_{i, j}$ mapping into $c_{j}$, and we must check what relations $\sum_{i, j} a_{i} b_{i, j}=0$ hold-i.e., we want a set of elements $\sum_{i, j} a_{i}\left[b_{i, j}\right] \in K$ generating all such relations. (Of course, care must be taken with vertices which map not only into $c_{j}$ !)

This may be verified locally, at each of the 13 vertex configurations appearing in Figure 2. In fact, it is not hard to see that there are only 9 types of essentially different vertices: $t, h, n, \tilde{n}, A, B, C, D$, and $E-c f . \S 3.1 .3$.

For example, if the vertex $c_{j} \in S_{n-1}$ is of type $A$ (mapping into $x$ and $y$, say), then it has the following vertices in $S_{n}$ mapping in

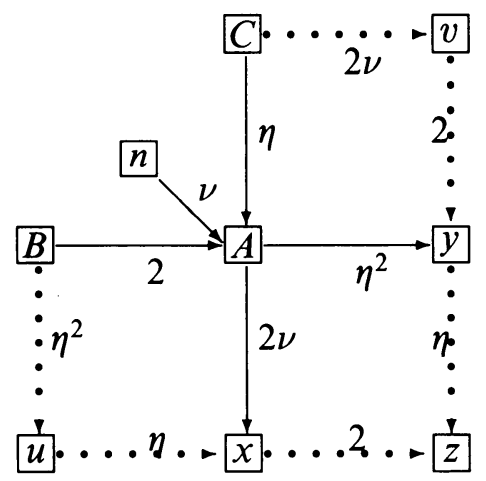

In $K$, we find $R$-module generators for the submodule of relations involving $2, \eta$, and $\nu$ are:

$$
\begin{array}{cc}
\eta[2], \quad\left(\nu^{2}\right)[2], & 2[\eta], \quad \nu[\eta], \quad \eta[\nu], \\
D=2[\nu]-\nu[2], & A=\left(\eta^{2}\right)[\eta]+(2 \nu)[2]
\end{array}
$$

(omitting $(4 \nu)[2]=2 A, 4[\nu]+\left(\eta^{2}\right)[\eta]=2 D+A, 8[\nu]=4 D+2 A,(2 \nu)[\nu]=$ $\left.\nu D+\left(\nu^{2}\right)[2]\right)$. Taking into account the neighboring vertices, we thus obtain the following (circled) vertices in $S_{n+1}$ - which are indeed included in Figure 2: 


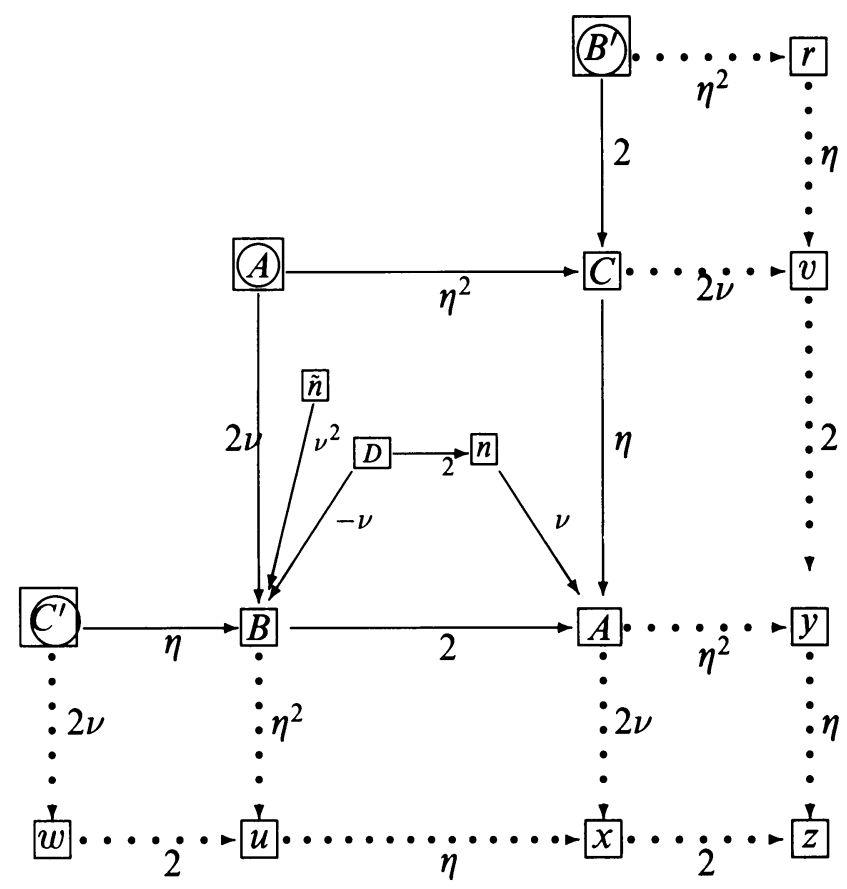

Similarly for the other 8 types of vertices.

3.1.6. An alternative description. If we merely wish to describe the sequence of sets generators for the resolution-i.e., $\left\{S_{n}\right\}_{n=0}^{\infty}$, without the differentials, or "attaching maps" $i_{n}: S_{n} \rightarrow R\left\langle S_{n-1}\right\rangle$, a simpler description is available:

Let $M$ be the free bigraded associative noncommutative $\mathbb{Z}$-algebra generated by $t, h, n, \tilde{n}, A, B, C, D, E$ in bidegrees $|t|=(0,1),|h|=(1,1)$, $|n|=(3,1),|\tilde{n}|=(6,1),|A|=|B|=|C|=|D|=(3,2)$, and $|E|=(9,2)$.

We define recursively a set $L$ of monomials in $M$ by

(a) $h, t, n \in L$

(b) $z y x \in L$ if $z y \in L$ and $y x \in\{h t, \tilde{n} t, t h, n h, \tilde{n} h, D h, E h, h n, A n$, $C n, t \tilde{n}, B \tilde{n}, n A, \tilde{n} A, A A, E A, B B, D B, t B, h C, C C, h D, \tilde{n} D, A D$, $C D, t E, B E\}$, where $z$ is possibly empty.

Then $L$ is isomorphic to the bigraded set $S_{* *}$ of generators for $P_{*}$ (where the homological degree is second).

(We are simply listing all the vertices in the graphical description of $P_{*}$ above, with the convention that the initial vertex in each square "multiplies on the right" (= acts on) the terminal vertex, and $i$ is omitted.)

A list of the elements of $L_{k, *}$ for $k \leq 6$ is given in Table 1.

\section{Application to the Hurewicz SPectral Sequence}

We now apply the calculation of $\S 3$ to the Hurewicz spectral sequence. 


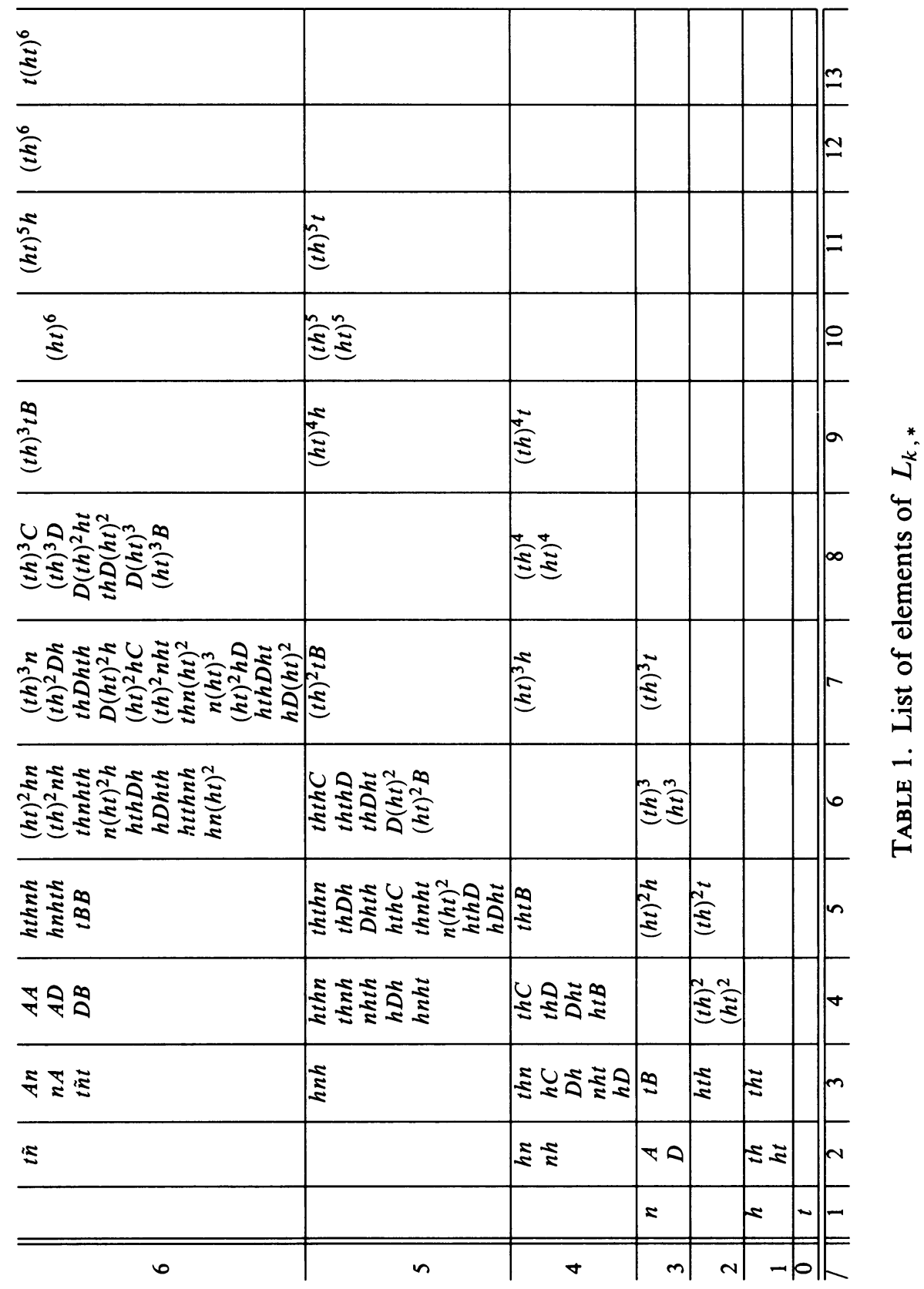


4.1. The bands of dependence. In particular, we wish to make use of the "bands of dependence" results of $[B 2, \S 4]$ ) to show why the simplified stable calculation of $\S 3.1$ is relevant even to the unstable spectral sequence:

Recall that the homotopy groups of any pointed connected space $\mathbf{X}$, together with the action of the primary homotopy operations on them (cf. [W, Chapter $\mathrm{X]}$ ), form a $\Pi$-algebra; and that for any abelian group $G$, we have a Hurewicz spectral sequence [B1] converging to $\tilde{H}_{*}(\mathbf{X} ; G)$, with $E^{2}$-term isomorphic to the derived functors of the indecomposables functor evaluated on $\pi_{*} \mathbf{X}$ (cf. [B1, $\S 3.3])$ :

$$
E_{n, t}^{2} \cong\left(L_{n}\left(Q_{t} \otimes G\right)\right)\left(\pi_{*} \mathbf{X}\right)
$$

Moreover, if $\mathbf{X}$ is $(r-1)$-connected $(r \geq 2)$, by [B2, Proposition 4.3] a certain band in the $E^{2}$-term depends only on the algebraic $k$-skeleton of $\pi_{*} \mathbf{X}$ : thus, for $n \geq 2(t-k)+1$, we find that $E_{n, t}^{2}$ is determined by $\left\{\pi_{i} \mathbf{X}\right\}_{i=r}^{k}$ and the action of the primary homotopy operations in this range.

4.1.1. The spectral sequence for $\mathbf{K}(\mathbb{Z} / 2, r)$. This implies that if $\mathbf{X}=\mathbf{K}(\mathbb{Z} / 2, r)$ and $r \geq 8$, we have $E_{n, t}^{2} \cong \operatorname{Tor}_{n, t}^{R}(\mathbb{Z} / 2, G)$ for $n \geq 2(t-(r+6))+1$ in this spectral sequence, (where $R=\pi^{(6)}$, as in $\S 2.3$ ).

Note that this implies more than the obvious fact that in the stable range, the nonabelian derived functors on the category of $\Pi$-algebras (here, the indecomposables functor $Q$ of $[\mathrm{B} 1, \S 2.2 .1]$ ) are isomorphic to the corresponding ordinary derived functors in the category of $\boldsymbol{\pi}$-modules (here, Tor).

4.1.2. Calculation of the $E^{2}$-term. Since the resolution of Proposition 3.1.5 is readily seen to be a minimal one, it actually provides a explicit description of the $E^{2}$-term in that band:

(i) For $G=\mathbb{Z} / 2$, the bigraded set $S_{* *}$ of $\S 3.1 .6$ serves as a $\mathbb{Z} / 2$-vector space basis for the $E^{2}$-term in the band in question.

(ii) For $G=\mathbb{Z}$, a basis for the $E^{2}$-term in the band may be obtained from $S_{* *}$ by omitting all monomials ending (on the right) in $t, B$, or $D$; all remaining monomials generate $\mathbb{Z} / 2$ summands.

4.2. The differentials. We now recall from $[S, \S 2]$ the construction of the simplicial space $\mathbf{V}_{\bullet}=\mathbf{V}_{\bullet}(\mathbf{X})$, used in $[B 1, \S 2.1]$ to construct the Hurewicz spectral sequence $\mathbf{X}$ (see also [B1, §6.2]).

4.2.1. The cotriple $\mathscr{V} . \mathrm{V}_{\text {. }}$ is constructed by means of a cotriple $\mathscr{V}$ on the category $\mathscr{T}_{*}$ of pointed connected spaces, which assigns to each $\mathbf{X}$ the space

$$
\mathscr{V}(\mathbf{X})=\bigvee_{k=1}^{\infty} \bigvee_{H_{o m_{*}}\left(\mathbf{S}^{k}, \mathbf{x}\right)} \mathbf{S}^{k} \cup \bigvee_{k=1}^{\infty} \bigvee_{H_{\text {om }}\left(\mathrm{e}^{k+1}, \mathbf{x}\right)} \mathbf{e}^{k+1},
$$

where the $(k+1)$-disc indexed by $F: \mathbf{e}^{k+1} \rightarrow \mathbf{X}$ is attached to the $k$-sphere indexed by $f=\left.F\right|_{\partial \mathbf{e}^{k+1}}$ (identifying $\partial \mathbf{e}^{k+1}$ with $\mathbf{S}^{k}$ ).

The simplicial space $\mathbf{V}_{\bullet}$ is then defined by setting $\mathbf{V}_{n}=\mathscr{V}^{n+1} \mathbf{X}$, with the usual face and degeneracy maps induced by the obvious counit and comultiplication (cf. [G, Appendix, §3]). 
4.2.2. The spectral sequence. By applying the singular chains functor we obtain a simplicial chain complex $\mathscr{S}_{*} \mathbf{V}_{\bullet}$, and taking alternating sums of the simplicial face maps yields a double chain complex. The spectral sequence is the usual one of a bicomplex, with $E_{n, t}^{2} \cong H_{n}^{h} \tilde{H}_{t}\left(\mathbf{V}_{\bullet} ; G\right)$, where $H_{*}^{h}$ is the homology in the external (simplicial) direction.

4.2.3. Notation for $\mathbf{V}_{\text {. }}$. A typical $k$-sphere in $\mathbf{V}_{n}$ may be then be indexed by a sequence of $n+1$ maps, $\mathbf{S}_{f_{0}, f_{1}, \ldots, f_{n}}^{k}$, with each $f_{i}: \mathbf{W}_{i} \rightarrow \mathbf{W}_{i-1}$ for some finite wedge of spheres (and disks) $\mathbf{W}_{i} \subseteq \mathbf{V}_{i}$ (with $\mathbf{W}_{i-1}=\mathbf{X}$ and $\mathbf{W}_{n}=\mathbf{S}^{k}$ ). Similarly for disks.

Note also that each element $\langle\gamma\rangle \in E_{n, k}^{2}$ of the Hurewicz spectral sequence for $\mathbf{X}$, represented by a generator $\gamma$ of $P_{n, k}$ as in $\S 2.3$, may be represented by a generator of $\tilde{H}_{k}\left(\mathbf{S}_{f_{0}, f_{1}, \ldots, f_{n}}^{k} ; G\right)$ as above by choosing representatives $f_{i}$ for the homotopy classes indexing $\gamma$. (See (1) in proof of Proposition 4.2.5 below for an example.)

We let $\left[\mathbf{S}^{k}\right]$ denote a singular $k$-simplex representing the generator of $\tilde{H}_{k}\left(\mathbf{S}^{k} ; \mathbb{Z}\right)$, for any sphere $\mathbf{S}^{k}$. Similarly, for any disk, let $\left[\mathbf{e}^{k+1}\right]$ represent a generator of $\tilde{H}_{k+1}\left(\mathbf{e}^{k+1}, \partial \mathbf{e}^{k+1} ; \mathbb{Z}\right)$.

4.2.4. More explicit notation. We can make this notation more explicit (though more cumbersome) by expressing the maps $f_{i}: \mathbf{W}_{i} \rightarrow \mathbf{W}_{i-1}$ as a sum of maps from the summands of $\mathbf{W}_{i}$.

For example, the element $\langle A\rangle \in E_{2, r+3}^{2}$ (cf. $\S 4.1 .2$ and Figure 1(a) of $\S 2.3 .1$ ) is represented by

$$
\left[\begin{array}{c}
r+3 \\
i,\left\{\begin{array}{l}
\eta \\
2
\end{array}\right\},\left(\eta^{2}\right) l_{1}+(2 \nu) l_{2}
\end{array}\right]
$$

where $\mathbf{W}_{1}=\mathbf{S}_{i, \eta}^{r+1} \vee \mathbf{S}_{i, 2}^{r}$, (indexed by the entries in the vertical array), and $l_{k}$ $(k=1,2)$ denotes the inclusion of the $k$ th sphere summand in $\mathbf{W}_{1}$.

4.2.5 Proposition. In the Hurewicz spectral sequence for any $\mathbf{X}$ and $G$, the differential $d^{2}\left[\mathbf{S}_{f_{0}, f_{1}, \ldots, f_{n}}^{k}\right](k \geq 2)$ may be represented by a sum

$$
\sum_{i=1}^{n-1}\left[\mathbf{S}_{f_{0}, \ldots, f_{i-2},\left\langle f_{i-1}, f_{i}, f_{i+1}\right\rangle, \sum f_{i+2}, \ldots, \sum f_{n}}^{k+1}\right]
$$

where $\left\langle f_{i-1}, f_{i}, f_{i+1}\right\rangle$ denotes some element in the Toda bracket.

Proof. Since $P_{*}$ is acyclic, each composition $f_{i-1} \circ f_{i}$ is nullhomotopic, so we may choose nullhomotopies $F_{i}: f_{i-1} \circ f_{i} \cong *$. Let $C f: \bigvee_{i} \mathrm{e}^{k_{i}+1} \rightarrow C \mathbf{W}$ denote the cone on a map $f: \bigvee_{i} \mathbf{S}^{k_{i}} \rightarrow \mathbf{W}$, where we identify $C \mathbf{S}^{k}$ with $\mathbf{e}^{k+1}$.

A straightforward calculation (as in $[\mathrm{B} 1, \S 6.3]$ ) then shows that $d^{2}\left[\mathbf{S}_{f_{0}, f_{1}, \ldots, f_{n}}^{k}\right]$ may be represented by

$$
\begin{aligned}
\sum_{0<j<i-1<n}(-1)^{i+j}\left(\left[\mathbf{e}_{f_{0}, \ldots, f_{j-1} \circ f_{j}, \ldots, F_{i}, C f_{i+1}, \ldots, C f_{n}}^{k+1}\right]\right. & \\
- & {\left.\left[\mathbf{e}_{f_{0}, \ldots, F_{j}, C f_{j+1}, \ldots, C\left(f_{i-1} \circ f_{i}\right), \ldots, C f_{n}}^{k+1}\right]\right) }
\end{aligned}
$$




$$
+\sum_{i=1}^{n-1}\left(\left[\mathbf{e}_{f_{0}, \ldots, f_{i-2}}^{k+1}, f_{i-1} \circ F_{i+1}, C f_{i+2}, \ldots\right]-\left[\mathbf{e}_{f_{0}, \ldots, f_{i-2}, F_{i} \circ C f_{i+1}, C f_{i+2}, \ldots}^{k+1}\right]\right),
$$

The first sum represents vanishing Toda brackets by [T, Proposition 1.2], while the second sum yields the Toda brackets we require.

We can use this proposition to calculate all $d^{2}$-differentials inside the "band of dependence" of $\S 4.1 .1$ :

4.2.6 Proposition. In the Hurewicz spectral sequence for $\mathbf{X}=\mathbf{K}(\mathbb{Z} / 2, r) \quad(r \geq 8)$ with coefficients in $\mathbb{Z}$ or $\mathbb{Z} / 2$, the differential $d^{2}$ may be evaluated on any allowable word $w \in L_{n, t}$ (cf. §4.1.2) for $n \geq 2(t-r)-6$, by adding the results of all applicable rules in the following list:

(i) $\quad[y(n h t h) x] \mapsto[y(t \tilde{n}) x]$,

(ii) $\quad[(h n h t) x] \mapsto[(t \tilde{n}) x]$,

(iii) $\quad[y($ thnh $) x] \mapsto[y(t \tilde{n}) x]$,

(iv) $\quad[y(t h n h) x] \mapsto[y(t \tilde{n}) x]$,

(v) $\quad[(h t h n) x] \mapsto[(t \tilde{n}) x]$,

(vi) $\left[y(t h)^{2} x\right] \mapsto\left\{\begin{array}{lll}{[A x]} & \text { if } y=\varnothing \quad \text { and } & x \neq t x^{\prime}, C, \\ {[y C x]} & \text { if } y=y^{\prime} h\end{array}\right.$ and $x \neq t x^{\prime}, C$,

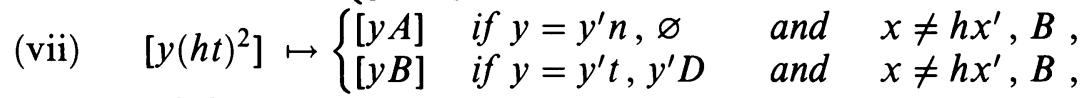

(viii) $[(h t h D) x] \mapsto[(A n+t \tilde{n} t) x]$,

(ix) $[y(D h t h)] \mapsto[y(n A)]$,

where $x, x^{\prime}, y, y^{\prime}$ are any allowable words (including the empty word $\varnothing$ ), subject to the stated restrictions.

\subsubsection{Examples.}

(a) $d^{2}\left[h n(h t)^{2}\right]=[h t \tilde{n} t]+[t \tilde{n} h t]+[h n A]$ by rules (i), (ii) and (vii).

(b) $d^{2}[\operatorname{thth} C]=0$ since none of the rules apply.

(c) $d^{2}\left[(t h)^{k+2} n\right]=\left[(t h)^{k} C n\right]$ since only rule (vi) applies.

(d) $d^{2}[h t h D h t h]=[$ Anhth $]+[t \tilde{n} t h t]+[h t h n A]$ by rules (viii) and (ix).

4.2.8. Proof of Proposition 4.2.6. The calculation is straightforward, using Proposition 4.2 .5 (which clearly implies the additivity). Since any word in the band in question has at most one of $n, A, B, C$, or $D$, the possible words are quite limited, and the only homotopy-theoretic information needed are the following Toda brackets:

$$
\langle 2, \eta, 2\rangle=\left\{\eta^{2}\right\}
$$$$
\langle\eta, 2, \eta\rangle=\{ \pm 2 \nu\}
$$$$
\langle\eta, \nu, \eta\rangle=\left\{\nu^{2}\right\}
$$

(cf. [T, Propositions 3.7, 1.4 and 5.12] respectively). We illustrate this by two examples:

(a) $d^{2}[t h t h]=[A]($ rule $(\mathrm{v}))$ : Note that $[t h t h]$ is represented by $\left[\mathbf{S}_{i, 2, \eta, 2, \eta}^{r+2}\right]$ (where we use stable homotopy classes to denote their representatives), and

$$
\begin{aligned}
d^{2}\left[\mathbf{S}_{i, 2, \eta, 2, \eta}^{r+2}\right] & =\left[\mathbf{S}_{\langle i, 2, \eta\rangle, 2, \eta}^{r+3}\right]+\left[\mathbf{S}_{i,\langle 2, \eta, 2\rangle, \eta}^{r+3}\right]+\left[\mathbf{S}_{i, 2,\langle\eta, 2, \eta\rangle}^{r+3}\right] \\
& =\left[\mathbf{S}_{i, \eta^{2}, \eta}^{r+3}\right]+\left[\mathbf{S}_{i, 2,2 \nu}^{r+3}\right]
\end{aligned}
$$


(The element $\left[\mathbf{S}_{\langle i, 2, \eta\rangle, 2, \eta}^{r+3}\right]$ vanishes in $E_{2, r+3}^{1}$, since it is indexed by a map $\mathbf{S}^{r+2} \rightarrow \mathbf{K}(\mathbb{Z} / 2, r)$.)

By evaluating the horizontal differential on $\left[\mathbf{S}_{i, \eta, \eta, \eta}^{r+3}\right] \in E_{3, r+3}^{1}$ (cf. [B1, $\S 6.2 .3])$, we see that $\left[\mathbf{S}_{i, \eta^{2}, \eta}^{r+3}\right]$ is homologous to $\left[\mathbf{S}_{i, \eta, \eta^{2}}^{r+3}\right]$; and finally $\left[\mathbf{S}_{i, \eta, \eta^{2}}^{r+3}\right]$ $+\left[\mathbf{S}_{i, 2,2 \nu}^{r+3}\right]$ is homologous to

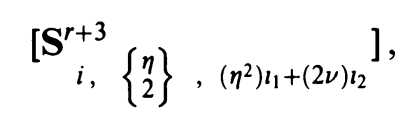

which is precisely $[A]$ in the notation of $\S 4.2 .4$ above.

(b) $d^{2}\left[t(h t)^{k+2}\right]=\left[t(h t)^{k} B\right]$ for $k \geq 0$ (rule (vii)): $\left[t(h t)^{k} B\right]$ is represented by the diagram

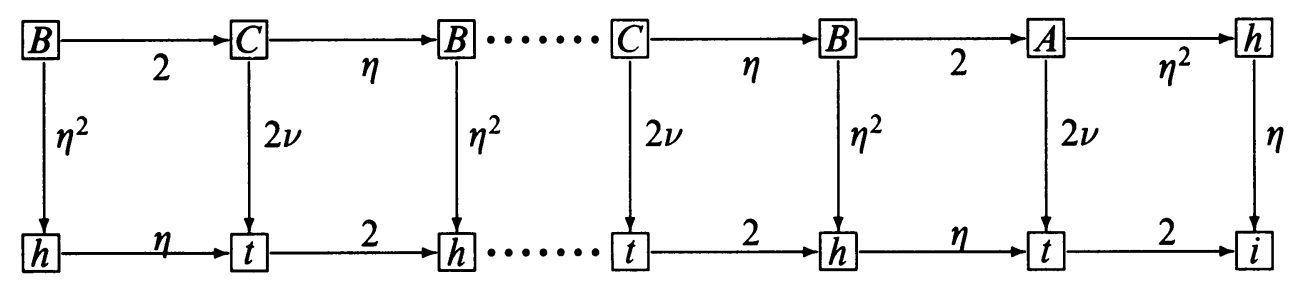

That is (in the notation of $\S 4.2 .4)$ by

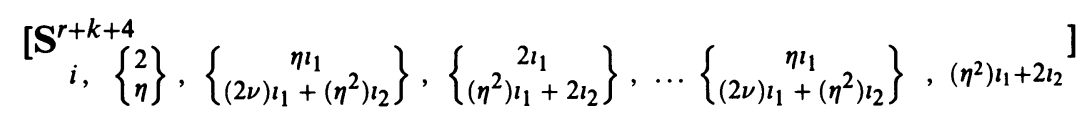

which may be shown as above to be homologous to

$$
\begin{array}{r}
{\left[\mathbf{S}_{i, 2, \eta, 2, \ldots, 2, \eta, \eta^{2}}^{r+k+4}\right]+\left[\mathbf{S}_{i, 2, \eta, 2, \ldots, 2,2 \nu, 2}^{r+k+4}\right]+\left[\mathbf{S}_{i, 2, \eta, 2, \ldots, \eta^{2}, \eta, 2}^{r+k+4}\right]} \\
+\cdots+\left[\mathbf{S}_{i, 2,2 \nu, 2, \ldots, \eta, 2, \eta, 2}^{r+k+4}\right]+\left[\mathbf{S}_{i, \eta, \eta^{2}, 2, \ldots, \eta, 2, \eta, 2}^{r+k+4}\right]
\end{array}
$$

(where the indices give all possible paths from the terminal (leftmost) $B$ to $i$ in the diagram above).

In (2), replace the last summand by the homologous $\left[\mathbf{S}_{i, \eta^{2}, \eta, 2, \ldots, \eta, 2, \eta, 2}^{r+k+4}\right]$ : this is precisely the result of applying Proposition 4.2.5 to $\left[t(h t)^{k+2}\right]$, using (1) above.

The remaining rules are shown similarly, and the only labor involved is in translating the results of applying Proposition 4.2.5 into a homologous allowable word (as in (a) above).

4.3. The Steenrod action. We can now calculate the $E^{\infty}$-term of the spectral sequence, in a certain range, and indicate how it is related to the Steenrod action.

4.3.1. The cohomology spectral sequence. Clearly the Hurewicz spectral sequence can be dualized at the $E^{0}$-level, that is, as a double complex (see $\S 4.2 .2)$ to yield a spectral sequence converging to $\tilde{H}^{*}(\mathbf{X} ; G)$, for any abelian group $G$. Taking $\mathbf{X}=\mathbf{K}(\mathbb{Z} / 2, r)$ and $G=\mathbb{Z} / 2$, the $E^{2}$-term we obtain is 
the vector space dual of that of $\S 4.1 .2$ above (in the band in question), and the differentials are dual to those of $\S 4.2$.

Since the spectral sequence (in the stable range) has as its $E^{2}$-term the $\mathbb{Z} / 2$ cohomology of the stable homotopy ring, and it converges to the Steenrod algebra, it deserves to be called the reverse Adams spectral sequence.

4.3.2. Multiplicative structure. As noted in [B1, §7.2], reverse Adams spectral sequences for spectra have long been known (and have been calculated in a range by Brayton Gray and Weh-Hsiung Lin, among others). In particular, the stable version of the cohomology spectral sequence, with $E_{2}^{*, *} \cong \operatorname{Ext} \pi(\mathbb{Z} / 2, \mathbb{Z} / 2)$, has as usual an algebra structure (cf. [A, $\S 4]$ or $[\mathrm{M}, \S 3])$. This agrees with the "product structure" defined by the operations (as in $\S 3.1 .6$ above), because both may be described in terms of Yoneda products (cf. [M, §2]).

On the other hand, the unstable cohomology spectral sequence has a different product, coming from the diagonal of $\mathbf{X}$. The operations we use allow us to represent the stable multiplication in the unstable spectral sequence (although in the example at hand our calculations are in the stable range, so we could have considered the stable spectral sequence only).

4.3.3. Extending the resolution. To calculate $\tilde{H}^{r+i}(\mathbf{K}(\mathbb{Z} / 2, r) ; \mathbb{Z} / 2)$ for $0 \leq i \leq$ 9 , (now with $r \geq 9$ ), we must slightly extend the resolution of $\S 3.1 .1$ to account for the new generator $\sigma \in \pi_{7}$ subject to the relations $(8 \sigma)[2], F \stackrel{\text { def }}{=} 2[\sigma]-\sigma[2]$.

We also have $\varepsilon \in \pi_{8}$, (or $\varepsilon, \bar{\nu} \in \pi_{r+8} \mathbf{S}^{r}$ if $r=8$ ), but since $\varepsilon \in\left\langle\eta, 2, \nu^{2}\right\rangle$ and $\bar{\nu} \in\langle\nu ; \eta, \nu\rangle$, they are killed by $d^{2}$. Thus we have

4.3.4 Proposition. The $E_{\infty}$-term for the reverse Adams spectral sequence for $\mathbf{K}(\mathbb{Z} / 2, r) \quad(r \geq 9)$, in degrees $\leq r+9$, may be identified (modulo higher filtrations) with the elements of $\tilde{H}^{*}(\mathbf{K}(\mathbb{Z} / 2, r) ; \mathbb{Z} / 2)$ as in Table 2.

Proof. Once we have applied $d_{2}$ from Proposition 4.2.5 to obtain $E_{3}$, a vectorspace dimension count shows this is in fact $E_{\infty}$ in the range in question. To identify the Steenrod structure, it is enough to identify the algebra generators $t, h, n$ and $D$, (using the stable multiplicative structure of $\S 4.3 .2$ )-which may be done by comparison with other spaces such as $\mathbf{K}(\mathbb{Z}, r), \mathbf{S}^{r} \cup_{\eta} \mathbf{e}^{r+1}$, $\mathbf{S}^{r} \cup_{\nu} \mathbf{e}^{r+1}$. (It is unclear which element survives in $E_{\infty}^{1,9}$, though the group is evidently $\mathbb{Z} / 2$.)

4.3.5. Remark on the Steenrod structure. We have chosen to use cohomology, and to write the elements of $\tilde{H}^{*}(\mathbf{K}(\mathbb{Z} / 2, r) ; \mathbb{Z} / 2)$ in terms of $S q^{1}, S q^{2}$, and $S q^{4}$ in order to underline the relation between the Steenrod action and the operators we have used to describe our resolution (and thus the $E^{2}$-term ).

Note that the relationship between the Steenrod action and our operators holds also unstably, and thus does not depend on the (stable) multiplicative structure of $\S 4.3 .2$; in fact there are obvious unstable analogues in a certain range). Unfortunately, this is not susceptible of any obvious generalization to a full $\boldsymbol{\pi}$-resolution of $\mathbb{Z} / 2$, even stably. 

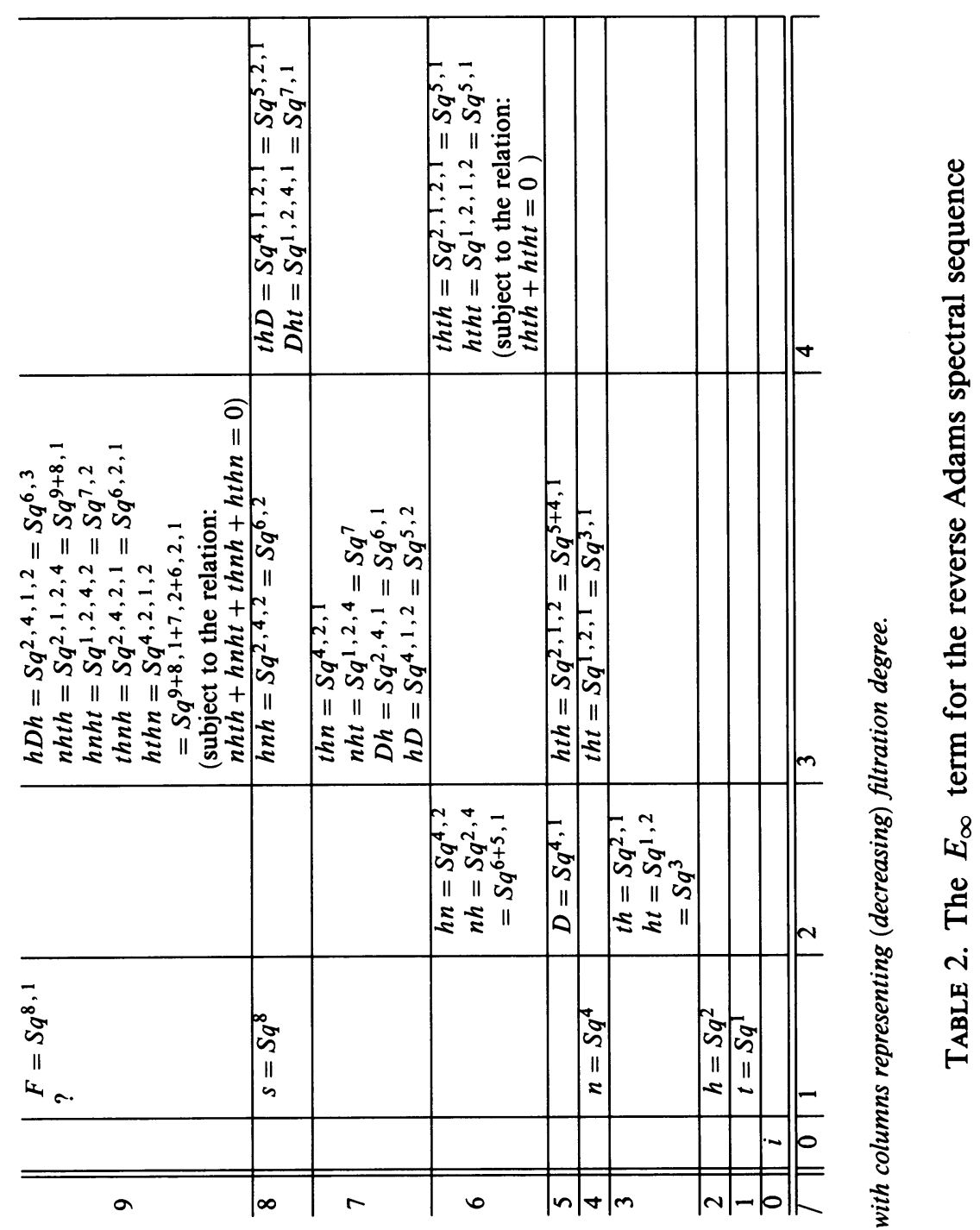
We can use Proposition 4.2.4 to obtain nonrealizability results for П-algebras, e.g.:

4.3.6 Proposition. There is no space $\mathbf{X}$ such that $\pi_{*} \mathbf{X} \cong \pi_{*} \mathbf{S}^{r} \otimes \mathbb{Z} / 2$ as $\Pi$ algebras (for $r \geq 6$ ).

Proof. The $\Pi$-algebra $M=\pi_{*} \mathbf{S}^{r} \otimes \mathbb{Z} / 2$ has a presentation $S_{0}=\{i\}, S_{1}=\{2[i]\}$ (cf. $\S 3.1 .1)$. In the stable band $(n \geq 2(t-r)+7)$ the generators of a minimal resolution of $M$ consist only of the words $t(h t)^{k} h^{\varepsilon} \quad(k \geq 0, \varepsilon=0,1)$, in the notation of $\S 3.1 .6$ and $\S 4.1 .2$.

If a space $\mathbf{X}$ with $\pi_{*} \mathbf{X} \cong M$ existed, the fundamental class in $\tilde{H}^{r}(\mathbf{X} ; \mathbb{Z} / 2) \cong$ $\mathbb{Z} / 2$ would represents a map $f: \mathbf{X} \rightarrow \mathbf{K}(\mathbb{Z} / 2, r)$ inducing an epimorphism of the $E^{2}$-term $\mathrm{s}$ in the cohomology spectral sequences .

Since there are no possible differentials on the band in question (for $\mathbf{X}$ ), and $f^{*}$ commutes with the squares as well as with our operations in $E^{\infty}$ of $\mathbf{K}(\mathbb{Z} / 2, r)$, we can calculate part of the Steenrod action on $\tilde{H}^{*}(\mathbf{X} ; \mathbb{Z} / 2) \cong E^{\infty}$ :

- $\tilde{H}^{r}(\mathbf{X} ; \mathbb{Z} / 2)=\mathbb{Z} / 2$, generated by $[i]$;

- $\tilde{H}^{r+2}(\mathbf{X} ; \mathbb{Z} / 2)=0$;

- $\tilde{H}^{r+6}(\mathbf{X} ; \mathbb{Z} / 2)=\mathbb{Z} / 2$, generated by $[$ thth $]=S q^{5,1}[i]$.

Since $S q^{2}[i]=0$, also $S q^{5,1}[i]=S q^{3,1,2}=0$, so that $\tilde{H}^{*}(\mathbf{X} ; \mathbb{Z} / 2)$ cannot even exist as an $\mathscr{A}$-module.

4.3.7. Note. A similar (unstable) calculation for $\tilde{H}^{*}(\mathbf{K}(\mathbb{Z} / 2, r) ; \mathbb{Z} / 2$ ) shows that the proposition holds in fact for $r \geq 3$. The analogous result for odd primes has been proven in [B4, Theorem 8.1].

\section{Projective dimension of $\pi$-MOdules}

As another application of the operations described in $\S 2$, we give a short proof of T. Y. Lin's result on the projective dimension of $\boldsymbol{\pi}$-modules, which we restate as follows:

5.1 Theorem (Lin, [L1, Theorem 1]): If $M$ is a $\boldsymbol{\pi}$-module of finite type which is not free, then $M$ has infinite projective dimension.

Proof. First note that any projective $\pi$-module is necessarily free (see [B3, Lemma 4.1] or [L2, Corollary 5.6]). Moreover, if $M$ is a $\pi$-module (of finite type) which is not free, this will also be true after localizing at $p$, for some prime $p$, so let $R=\pi_{(p)}$. It suffices to show that any map $f: P \rightarrow M$ from a free $R$-module onto one which is not free supports some nontrivial operation (in the sense of $\S 2.2$ )-which means in particular that $\operatorname{ker}(f)$ is not free.

Let $s \geq 0$ be the first degree in which $M$ is not free, and write

$$
P=\bigoplus_{i=1}^{L} R\left\langle a_{i}\right\rangle \oplus \bigoplus_{j=1}^{M} R\left\langle b_{j}\right\rangle \oplus \text { (higher degrees) }
$$

where $\left|a_{i}\right|<s$ and $\left|b_{j}\right|=s$. 
We may assume $P$ is minimal among free modules mapping onto $M$, so $\operatorname{ker}(f)$ is $(s-1)$-connected. Let $K=\operatorname{ker}(f)_{s} \neq 0$, where

$$
K \cong \bigoplus_{k=1}^{m} \mathbb{Z}_{(p)}\left\langle c_{k}\right\rangle \oplus \bigoplus_{l=1}^{n}\left(\mathbb{Z} / p^{r_{l}}\right)\left\langle d_{l}\right\rangle
$$

(a) If $n \neq 0$, let $\alpha_{1} \in R_{2 p-3}$ be the first element of order $p$ in $R$; then each cyclic generator $d_{l}$ supports an infinite "spike" of operations

$$
p\left[\alpha_{1}\left[\ldots\left[p\left[\alpha_{1}\left[p^{r_{l}}\left[d_{l}\right]\right]\right]\right] \ldots\right]\right]
$$

(analogous to sequence of elements $(t h)^{k} t^{\varepsilon}$ just above the vanishing line in Table 1). This shows $\operatorname{Tor}_{*}^{R}(M, \mathbb{Z} / p)$ has nonzero elements in each homological dimension.

(b) Next assume $n=0$ and $K \cong \bigoplus_{k=1}^{m} \mathbb{Z}_{(p)}\left\langle c_{k}\right\rangle$. Each $c_{k} \in P_{s}$ has the form

$$
c_{k}=\sum_{i=1}^{L} \zeta_{i, k}\left(a_{i}\right)+\sum_{j=1}^{M} n_{j, k}\left(b_{j}\right)
$$

where $n_{j, k} \in R_{0}=\mathbb{Z}_{(p)}$ (not all zero) and $\zeta_{i, k} \in R_{\geq 1}$.

By the minimality of $P$ we may assume $p \mid n_{j, k}$ for all $j$. Since $R_{\geq 1}$ is all $p$-torsion, there is an $r \geq 1$ such that $p^{r}\left(\zeta_{i, k}\right)=0$ for all $i$. Therefore, if $\alpha \in R$ is an element of order $p^{r+1}$, the fact that

$$
\left(p^{r} \alpha\right) \zeta_{i, k}=0=\left(p^{r} \alpha\right) n_{j, k}
$$

shows that $p^{r} \alpha$ acts on $c_{k}$ (in the sense of $\S 2.2 .2$ ), so $\operatorname{ker}(f) \not \bigoplus_{k=1}^{m} R\left\langle c_{k}\right\rangle-$ and thus is not free.

But then $K$ supports some operation of least degree (necessarily positive), which yields a nontrivial element in $\operatorname{Tor}_{*}^{R}(M, \mathbb{Z} / p)$.

This completes the induction step, and shows that $\operatorname{Tor}_{*}^{R}(M, \mathbb{Z} / p)$ actually has nontrivial elements in each homological dimension-so that $M$ has infinite projective dimension.

5.2. An unstable version. An unstable version of this theorem has been proved in [B3, Theorem 4.3]. This states that any abelian $\Pi$-algebra has projective dimension 0,1 , or $\infty$, and if it is simply connected of finite type, it has projective dimension 0 or $\infty$.

The proof there also shows what changes have to be made in the stable case if the $\pi$-module $M$ is not assumed to be of finite type.

\section{REFERENCES}

[A] J. F. Adams, On the structure and applications of the Steenrod algebra, Comment Math. Helv. 32 (1958), 180-214.

[B1] D. Blanc, A Hurewicz spectral sequence for homology, Trans. Amer. Math. Soc. 318 (1990), 335-354.

[B2] _ _ Derived functors of graded algebras, J. Pure Appl. Algebra 64 (1990), 239-262. 
[B3] _ Abelian П-algebras and their projective dimension, Algebraic Topology-Oaxtepec 1991 (M. C. Tangora, ed.), Contemp. Math., vol. 146, Amer. Math. Soc., Providence, R.I., 1993, pp. 39-48.

[B4] _ Higher homotopy operations and the realizability of homotopy groups, Proc. London Math. Soc. (to appear).

[G] R. Godement, Topologie algébrique et théorie des faisceaux, Actualités Sci. Indust. 1252, Publ. Inst. Math. Univ. Strasbourg XIII, Hermann, Paris, 1964.

[L1] T. Y. Lin, Homological algebra of stable homotopy ring $\boldsymbol{\pi}_{*}$ of spheres, Pacific J. Math. 38 (1971), 117-142.

[L2] _ Homological dimensions of stable homotopy modules and their geometric characterizations, Trans. Amer. Math. Soc. 172 (1972), 473-490.

[M] R. M. F. Moss, On the composition pairing of Adams spectral sequences, Proc. London Math. Soc. (3) 18 (1968), 179-192.

[T] H. Toda, Composition methods in the homotopy groups of spheres, Princeton Univ. Press, Princeton, N. J., 1962.

[S] C. Stover, A Van Kampen spectral sequence for higher homotopy groups, Topology 29 (1990), 9-26.

[W] G. W. Whitehead, Elements of homotopy theory, Graduate Texts in Math. No. 61, SpringerVerlag, Berlin and New York, 1971.

Department of Mathematics, Northwestern University, Evanston, Illinois 60208 Current address: Department of Mathematics and Computer Science, Haifa University, Haifa, Israel

E-mail address: blanc@mathcs2.haifa.ac.il 